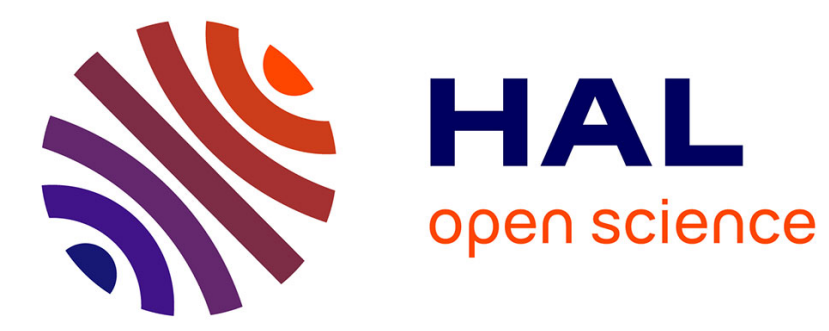

\title{
Multiple spatially localized dynamical states in friction-excited oscillator chains
}

\author{
A. Papangelo, Norbert Hoffmann, Aurelien Grolet, Merten Stender, M.
}

Ciavarella

\section{To cite this version:}

A. Papangelo, Norbert Hoffmann, Aurelien Grolet, Merten Stender, M. Ciavarella. Multiple spatially localized dynamical states in friction-excited oscillator chains. Journal of Sound and Vibration, 2018, 417, pp.56-64. 10.1016/j.jsv.2017.11.056 . hal-02121542

\section{HAL Id: hal-02121542 \\ https://hal.science/hal-02121542}

Submitted on 14 Dec 2019

HAL is a multi-disciplinary open access archive for the deposit and dissemination of scientific research documents, whether they are published or not. The documents may come from teaching and research institutions in France or abroad, or from public or private research centers.
L'archive ouverte pluridisciplinaire HAL, est destinée au dépôt et à la diffusion de documents scientifiques de niveau recherche, publiés ou non, émanant des établissements d'enseignement et de recherche français ou étrangers, des laboratoires publics ou privés. 


\section{Spatially localized multiple dynamical states in friction-excited oscillator chains}

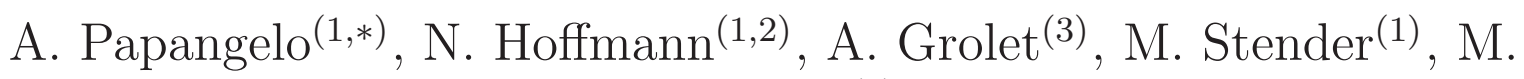
Ciavarella $^{(4)}$

(1) Hamburg University of Technology, Department of Mechanical Engineering, Am Schwarzenberg-Campus 1, 21073 Hamburg, Germany

(2) Imperial College London, Exhibition Road, London SWr 2AZ , UK

(3) Arts et Métiers ParisTech, Department of Mechanical Engineering, 8 Boulevard Louis XIV, 59000 Lille, France

(4) Politecnico di BARI. DMMM dept. V Gentile 182, 70126 Bari.

${ }^{*}$ email: antonio.papangelo@poliba.it

\section{Abstract}

Friction-induced vibrations are known to affect many engineering applications. Here, we study a chain of friction-excited oscillators with nearest neighbor elastic coupling. The excitation is provided by a moving belt which moves at a certain velocity $v_{d}$ while friction is modelled with an exponentially decaying friction law. It is shown that in a certain range of driving velocities, multiple stable spatially localized solutions exist whose dynamical behavior (i.e. regular or irregular) depends on the number of oscillators involved in the vibration. The classical non-repeatability of friction-induced vibration problems can be interpreted in light of those multiple stable dynamical states. These states are found within a "snaking-like" bifurcation pattern. Contrary to the classical Anderson localization phenomenon, here the underlying linear system is perfectly homogeneous and localization is solely triggered by the friction nonlinearity.

Keywords: nonlinear vibrations, localization, dry friction, friction-induced vibrations 


\section{Introduction}

Many engineering applications make use of frictional damping to dissipate energy. In particular, dry friction is commonly used as a cheap and convenient solution even in very hostile environments, as those where turbine blade rotors operate [1-5]. Also, many general engineering structures are assembled using different kinds of joints, which always introduce interfaces and thus dissipation (and damping) [6-11]. Nowadays many researchers are putting their effort in trying to control (or reduce) frictional resistance [1215]. In turn, vibrations induced by friction are experienced in many systems too, like in friction brakes, where they generate discomfort and noise [16-19]. Similar phenomena have different names depending on the context: "chatter" in workpiece manufacturing, "brake squeal" and "groan" in automotive and railways industries, and other industrial problems are related to the interplay of frictional contact and system (nonlinear) dynamics [18, 19, 22-24]. Many lumped models have been introduced over time which have tried to capture the underlying phenomena that cause friction-excited stick-slip limit cycles for example, there is a large literature on the classical mass-on-moving-belt system [25-28]. It has been shown that if at the mass-belt interface, a velocity exponential decaying friction law is assumed, the oscillator undergoes a subcritical Hopf bifurcations [29, 30] which naturally implies a region of the control parameter (i.e. the belt velocity) where the system is bistable (i.e., has two stable solutions), and can approach a Steady-Sliding state (SS) with no vibrations, or a High Amplitude Limit Cycle (HALC) [31].

The present work is devoted to study nonlinear localization phenomena in frictional systems particularly in the case where many elementary cells (the "unit cell") can be recognized, which are assembled together in a form of a chain of weakly coupled elements (see for example the high speed train brake pad in Fig. 1). The possibility for each of them to experience a twofold equilibrium opens the possibility of multi-stable spatially localized equilibrium states when these single components are elastically weakly linked together, allowing mutual interactions. Vakakis has studied weakly coupled chains extensively (see [32]) with various nonlinearities and forcing concepts, showing "nonlinear normal modes" (NNM) which localize in space and are a property of "weakly coupled" chains [32]. Recently, localized vibrating states have been observed in similar systems (both conservative and selfexcited) with geometric nonlinearities [33-35] or nonlinear damping [36]. In the bifurcation diagram, patterns similar to the "snaking bifurcations" are 
encountered, which have been studied in different physics fields, from fluid dynamics [37-39] to optics [37].

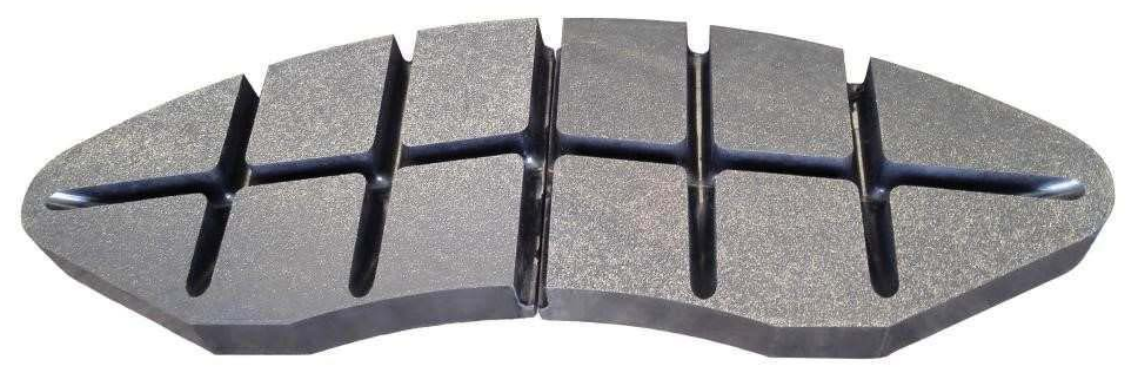

Figure 1 - A typical design for a high speed train brake pad ${ }^{1}$. Deep grooves subdivide the lining material in multiple sectors.

In this study, we will consider a chain of linear oscillators placed on a frictional moving belt driven at a certain velocity. Friction will be described using an exponentially decaying friction law. We remark that the underlying linear system is perfectly homogeneous and with periodic boundary condition (i.e., the first and the last oscillator are also elastically linked), thus if localization arises, it is from the friction nonlinearity. This happens in contrast to the classical Anderson localization [40], which is a linear phenomenon well known in mechanical engineering under the name of "mistuning" [41, 42].

The effect of the driving velocity and of the initial conditions is shown to affect the localization pattern.

\section{The model: a chain of nonlinear oscillators}

The model is an extension of the classical mass-on-moving-belt model. We consider a chain of $N$ linear oscillators (each oscillator has one degree of freedom, see Fig. 2) which are elastically weakly coupled via a linear spring $k_{c}$. Each oscillator has mass $m$, stiffness $k$, viscous damping coefficient $c$ and is pressed by a constant normal force $P$ against the belt. The position of

\footnotetext{
${ }^{1}$ (source: http://www.huawu-brakes.com/products_detail/productId=147.html)
} 
each oscillator is denoted with $x$, and a prime indicates differentiation with respect to time $\frac{d \square}{d t}=\square^{\prime}$.

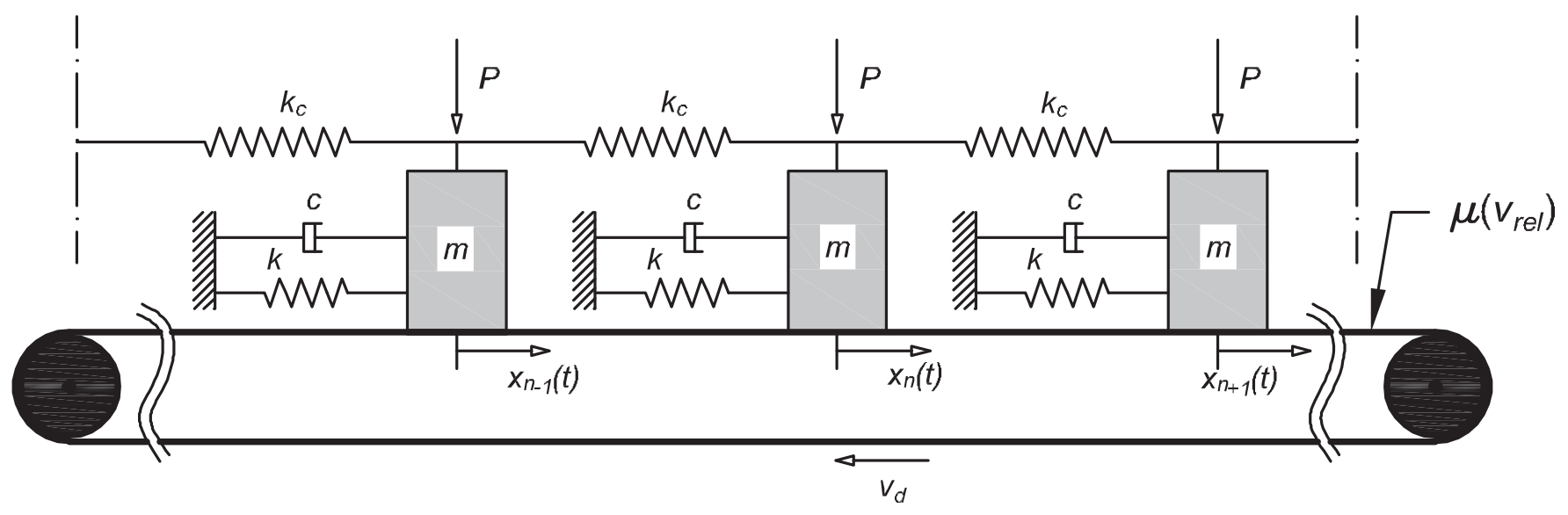

Figure 2. Geometrical model. It consists of $N$ weakly coupled nonlinear oscillators pressed against a frictional belt.

Friction between the oscillators and the belt is described using an exponential decaying friction law of the relative velocity $v_{r e l}=x^{\prime}-v_{d}$

$$
\mu\left(v_{r e l}\right)=\mu_{d}+\left(\mu_{s t}-\mu_{d}\right) \exp \left(-\frac{\left|v_{r e l}\right|}{v_{0}}\right)
$$

where $v_{0}$ is a reference velocity, $\mu(0)=\mu_{s t}$ is the static friction coefficient and $\mu\left(v_{r e l} \rightarrow+\infty\right)=\mu_{d}$ is the dynamic friction coefficient, with $\mu_{s t}>\mu_{d}$. Thus, the friction force is

$$
\begin{cases}F=-P \mu\left(v_{\text {rel }}\right) \operatorname{sign}\left(v_{\text {rel }}\right) & v_{\text {rel }} \neq 0 \\ |F|<\mu_{s t} P & v_{\text {rel }}=0\end{cases}
$$

where $v_{r e l}$ is the relative velocity and $\operatorname{sign}(\bullet)$ is the sign function.

The equilibrium equation for the $n$-th oscillator $(n=1,2, \ldots, N)$ reads

$$
m x_{n}^{\prime \prime}+c x_{n}^{\prime}+k x_{n}+k_{c}\left(2 x_{n}-x_{n+1}-x_{n-1}\right)=F\left(v_{r e l}\right),
$$

To obtain a dimensionless formulation of (3) we introduce the following quantities

$$
\omega_{n}=\sqrt{k / m}, \quad x_{0}=P / k, \quad \eta_{c}=k_{c} / k, \quad \xi=\frac{c}{2 \sqrt{k m}}, \quad \tau=\omega_{n} t
$$


where $\omega_{n}$ is the natural frequency of the single oscillator, $x_{0}$ is a reference displacement, $\eta_{c}$ is the dimensionless elastic coupling parameter, $\xi$ is the damping ratio and $\tau$ is a dimensionless time. Substituting $\frac{d \square}{d t}$ with $\omega_{n} \frac{d \square}{d \tau}=$ $\omega_{n} \square$ and dividing (3) by $P$, we obtain

$$
\ddot{\widetilde{x}}_{n}+2 \xi \dot{\widetilde{x}}_{n}+\widetilde{x}_{n}+\eta_{c}\left(2 \widetilde{x}_{n}-\widetilde{x}_{n+1}-\widetilde{x}_{n-1}\right)=\widetilde{F}\left(\widetilde{v}_{r e l}\right),
$$

where a tilde superposed indicates a dimensionless quantity, e.g.

$$
\widetilde{x}=\frac{x}{x_{0}}, \quad \widetilde{v}_{r e l}=\frac{v_{r e l}}{x_{0} \omega_{n}}, \quad \widetilde{F}=\frac{F}{P}
$$

Notice that in (5) the differentiation is carried out with respect to the dimensionless time $\tau$.

\section{Results}

\subsection{Numerical Integration}

A system with $N=20$ oscillators has been chosen for the numerical investigation which yields $2 N$ first order Ordinary Differential Equations (ODEs). The system of ODEs has been integrated using MATLAB ${ }^{\circledR}$, in particular the function ode23t. This ODE solver use the trapezoidal rule with a "free" interpolant, has no numerical damping and is recommended for moderately stiff problems. The implementation of the friction force required particular attention due to non-smoothness. Two approaches have been tested: smoothing of the signum function and the implementation of the switch model ([43]). The best results in term of calculation speed and stability have been obtained using the switch model, where it was assumed that the oscillator sticks to the belt if $\left|\widetilde{v}_{r e l}\right|<10^{-4}$ (see [43] for more details).

\subsection{Multiple localized vibration states}

Recently the authors have studied a one degree of freedom mass-onmoving-belt system (see [31] for more details). It has been found that for the following parameter set

$$
\xi=0.05, \quad \mu_{d}=0.5, \quad \mu_{s t} / \mu_{d}=2, \quad v_{0}=0.5
$$

the single oscillator experiences a subcritical Hopf bifurcation (see Fig. 3 (a)), and thus bistability (i.e. there exist two stable solutions for the same 
parameter set), for the range of driving velocity $1.1 \lesssim \widetilde{v}_{d} \lesssim 1.8$. In between the two stable solutions (Fig. 3 (a), black dots) an unstable limit cycle exists (Fig. 3 (a), dashed line). Here, we assume the same set of parameters, but we add a small elastic coupling term $\eta_{c}=0.01$. We integrated the equations of motion starting from different initial conditions. At $\tau=0$ we assume $\widetilde{x}_{n}(0)=0$ for all the oscillators while varying the initial displacement $\widetilde{x}_{n}(0)$

$$
\begin{array}{ll}
\widetilde{x}_{n}(0)=\widetilde{F}\left(-\widetilde{v}_{d}\right) & n \text {-th oscillator is "not excited" } \\
\widetilde{x}_{n}(0)=2(-1)^{n}+\widetilde{F}\left(-\widetilde{v}_{d}\right) & n \text {-th oscillator is "excited" }
\end{array}
$$

Notice that in the SS solution all the oscillators have a constant displacement $\widetilde{x}_{n}=\widetilde{F}\left(-\widetilde{v}_{d}\right)$. The solution found with the time integration technique are plotted in the bifurcation diagram shown in Fig. 3 (b): naturally, they are all stable. For characterizing each state of the system we have computed the vibration amplitude of each oscillator after the transient (assuming the transient is ended after 10000 dimensionless time units). A scalar quantity $\widetilde{E}$, with the dimension of an energy, has been defined as half of the sum of the squared vibration amplitude $\widetilde{A}_{i}$ of each oscillator.

$$
\widetilde{E}=\frac{1}{2} \sum_{i=1}^{N} \widetilde{A}_{i}^{2}
$$

In the following we will refer to $\widetilde{E}$ as a measure of the "vibration energy of the system", but we warn the reader that $\widetilde{E}$ does not correspond to the actual time varying vibration energy, as, in general, the oscillators vibrate with a varying phase difference.

The bifurcation diagram Fig. 3 (b) shows that multiple stable equilibrium solutions exist in the range $1.1 \lesssim \widetilde{v}_{d} \lesssim 1.8$, where the single oscillator is known to be bistable. The upper branch in Fig. 3 (b) represents the state where all the chain is excited and each oscillator experiences a stick-slip limit cycle, which is stable for $\widetilde{v}_{d} \lesssim 1.8$, while the lowest branch with null energy is stable for $\widetilde{v}_{d} \gtrsim 1.1$ and corresponds to the full sliding solution with $\widetilde{A}_{i}=0(i=1,2, \ldots, N)$, i.e. where each oscillator is motionless. In between the upper and lower branches, multiple spatially localized vibrating states exist whose energy content lies in between the upper and lower branches. In the regions delimited by dashed vertical lines (Fig. 3 (b)), propagating front solutions have been found, where stick-slip limit cycle states, instead of 
remaining spatially localized, enter in the full sliding domain or vice versa. The characterization of these propagating states goes beyond the scope of the present investigation.
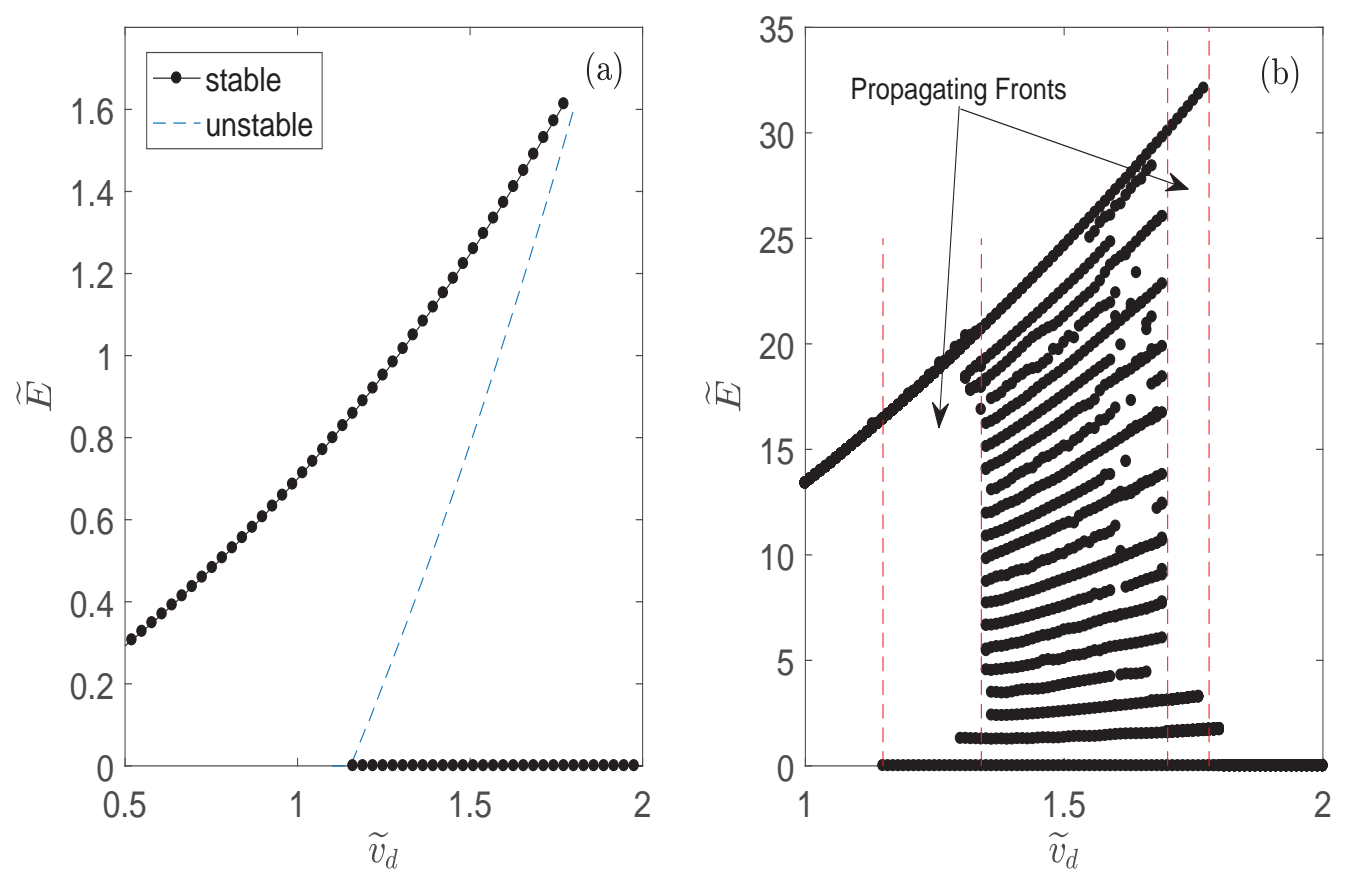

Figure 3 - Bifurcation diagram: the vibrational energy of the system is plotted versus the driving velocity of the belt in dimensionless form, (a) for the single oscillator (b) for the oscillator chain. In the panel (b) multiple stable equilibrium solutions can be seen in the range of diving velocity $1.1 \lesssim \widetilde{v}_{d} \lesssim 1.8$. The intermediate "energy levels" represents spatially localized states (see Fig. 4). The red dashed vertical lines indicate regions where propagating front solution have been observed.

Figure 4 clearly shows how the vibration is confined in space. Each circle represents one oscillator, with the circle radius proportional to the vibration amplitude. In Fig. 4, solutions for $N=20$ have been plotted for a fixed belt velocity $\widetilde{v}_{d}=1.5$ which corresponds to the "energy steps" encountered while climbing the bifurcation diagram from the bottom to the top. The higher the energy, the higher the number of oscillators involved in the vibration from 1 to 20. This picture strongly resembles the snaking bifurcation patterns observed in physics fields [39]. 


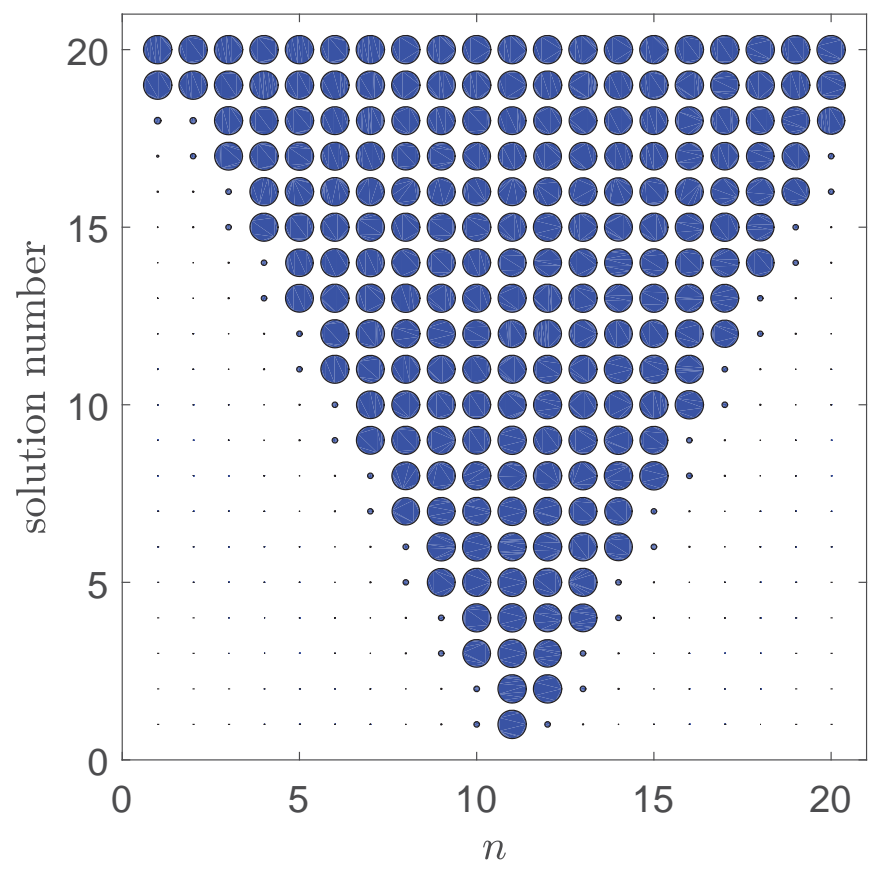

Figure 4 - Localized states obtained at $\widetilde{v}_{d}=1.5$ in Fig. 3. Each circle represents one oscillator, with the radius proportional to the amplitude of the vibration.

Typical time series are reported in Fig 5 in dimensionless notation: (a) displacement vs time, (b) phase plot velocity vs displacement (c) velocity vs time. The time window has been chosen to show the transient phase while the phase plot (Fig. 5 (c)) clearly shows the stick phase when the velocity of the oscillators equals the driving velocity of the belt, i.e. $\widetilde{v}_{d}=1.5$ in this particular case. Despite this case has only two oscillators which lie on the High Amplitude Limit Cycle (HALC) it can be seen that the vibration amplitude stabilizes quite soon to its steady state value, while the phase shift among the oscillators changes over time. This is a peculiar feature of the system under study; in fact, in the following, we will concentrate our attention on the relative phases rather then on the vibration amplitude. 

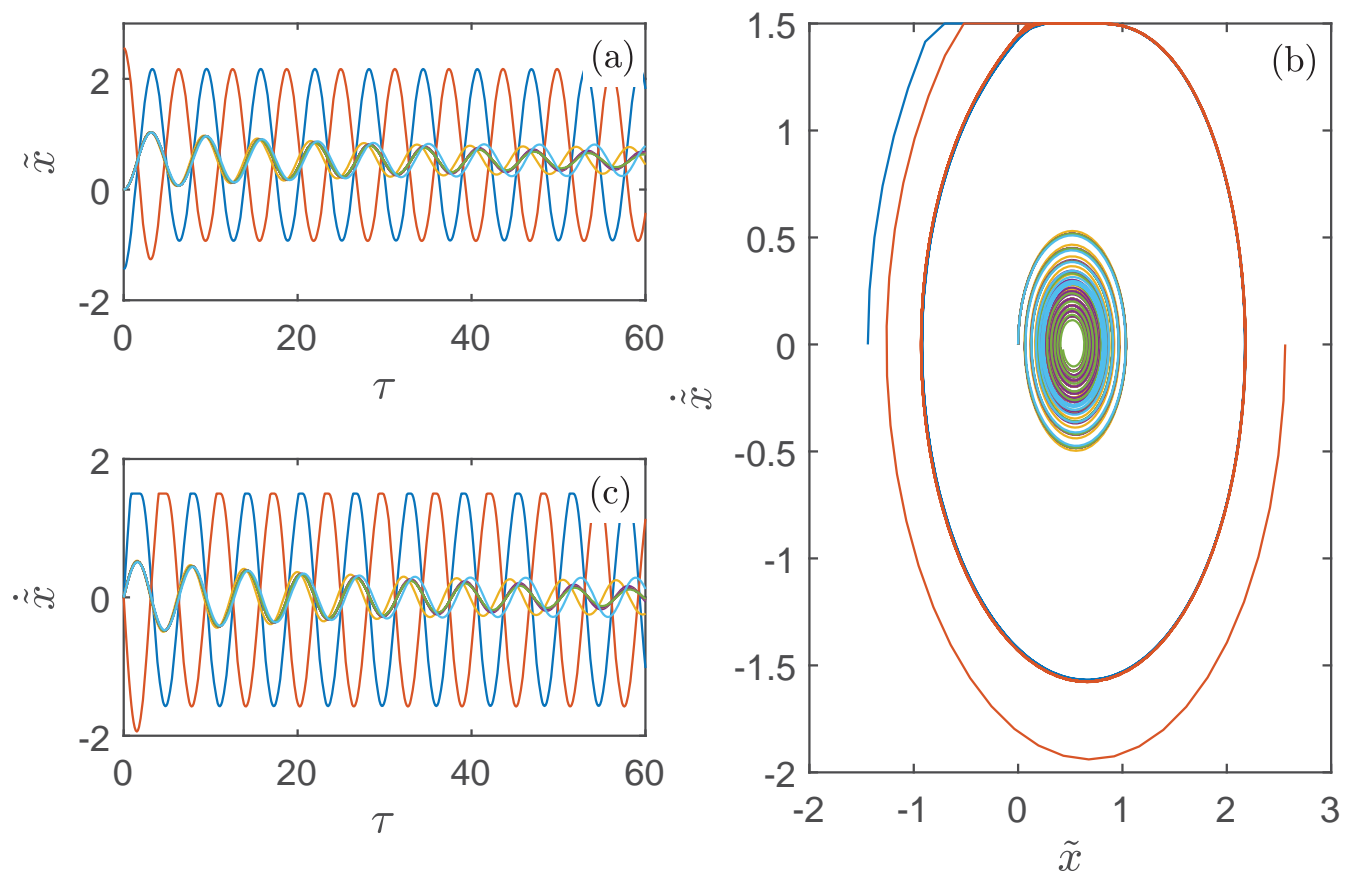

Figure 5 - (a) Displacement versus time, (b) phase plot, (c) velocity versus time of the 20 oscillators for $\widetilde{v}_{d}=1.5$. Different colors have been used to distinguish the trajectory of each oscillator.

\subsection{Dynamics of the localized states}

We have seen in the previous paragraphs that spatially localized vibrating states develops in the range of driving velocity $1.1 \lesssim \widetilde{v}_{d} \lesssim 1.8$. Here, we shall characterize the dynamical behavior of those localized states. As seen before, the vibration amplitude settles quite soon to a certain value, while the oscillators adjust their relative phases. Thus, we look at the relative phase between one oscillator taken as a reference (the $11^{\text {th }}$ ) and the others. The relative phase has been computed as a time delay $\phi$ between correspondent peaks, thus for the i-th oscillator $\phi_{i}=\tau_{\text {peak }, i}-\tau_{\text {peak }, 11}$. We select the $11^{\text {th }}$ oscillator because it was always excited and exhibits HALC in all the solutions we plotted (see Fig. 4). Fig. 6 shows, in the subplots from (a) to (i), the time delay $\phi$ of each oscillator which is on the HALC respectively for the solutions labeled by the number $[1,2,5,8,9,12,13,19,20]$ in Fig. 4 . We first notice that the equilibrium equations have been integrated for a very long time window, i.e. $\tau \in[0,15000]$, if compared with the duration of a single oscillation of the single oscillator. In fact, within the time span considered there are roughly 2400 complete oscillations, which last about $2 \pi$, which is the natural period of the isolated single oscillator. This was needed as the 
systems evolves very slowly to its steady-state (when the latter exists). In general when less oscillators are involved in the vibration we obtain periodic states. Looking at Fig. 6 this is the case of subplots (a,b,d,h,i) corresponding to the solutions $(1,2,8,19,20)$ in Fig. 4. In Fig. 6 (i) all the 20 oscillators are vibrating out of phase (this would be the last mode of the linearized system) with a time delay $\phi=0$ or $\pi$. In some cases a very long transient leads to an irregular motion, like in Fig. 6 (c-e-f-g) which correspond to solutions $(5,9,12,13)$ in Fig. 4. The irregular dynamical behaviour ${ }^{2}$ can be better appreciated looking at the three-dimensional phase plots shown in Fig. 7 for the same solutions shown in Fig. 6. In drawing the phase plots, we consider the time series of a simulation and remove the transient phase, assumed to be completed after 10000 dimensionless time units, thus we plot on the same graph the displacement of the oscillators $(11,12,13)$ for the time span $\tau \in[1,1.5] \times 10^{4}$. The two figures (Fig. 6-7) favorably compare: when $\phi$ remains constant among the oscillators a smooth periodic orbit is obtained (compare subplots (a-b-d-h-i) of Fig. 6-7), on the other hand when $\phi$ changes without reaching any asymptotic value the phase plots look tangled and irregular (compare subplots (c-e-f-g) of Fig. 6-7).

\footnotetext{
${ }^{2}$ Notice that the irregular states found could be chaotic states, as it has been shown in other dynamical systems subjected to dry friction [44-47].
} 

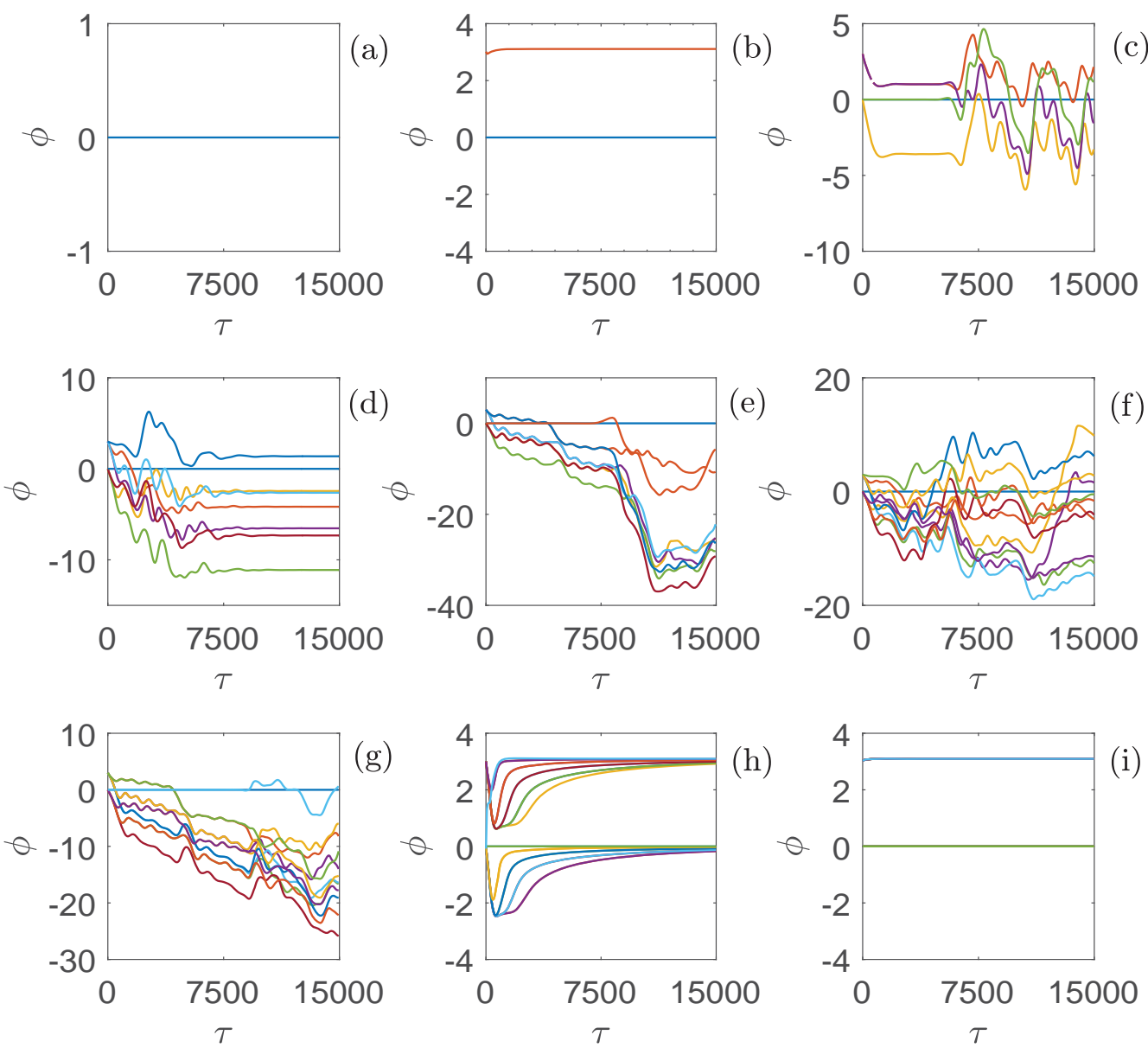

Figure 6 - From (a) to (i), time delay with respect to the $11^{\text {th }}$ oscillator $\phi_{i}=\tau_{\text {peak }, i}-\tau_{\text {peak }, 11}$ for the solutions $[1,2,5,8,9,12,13,19,20]$ reported in Fig. 4. Notice that both periodic and irregular states were found depending on the initial conditions. 

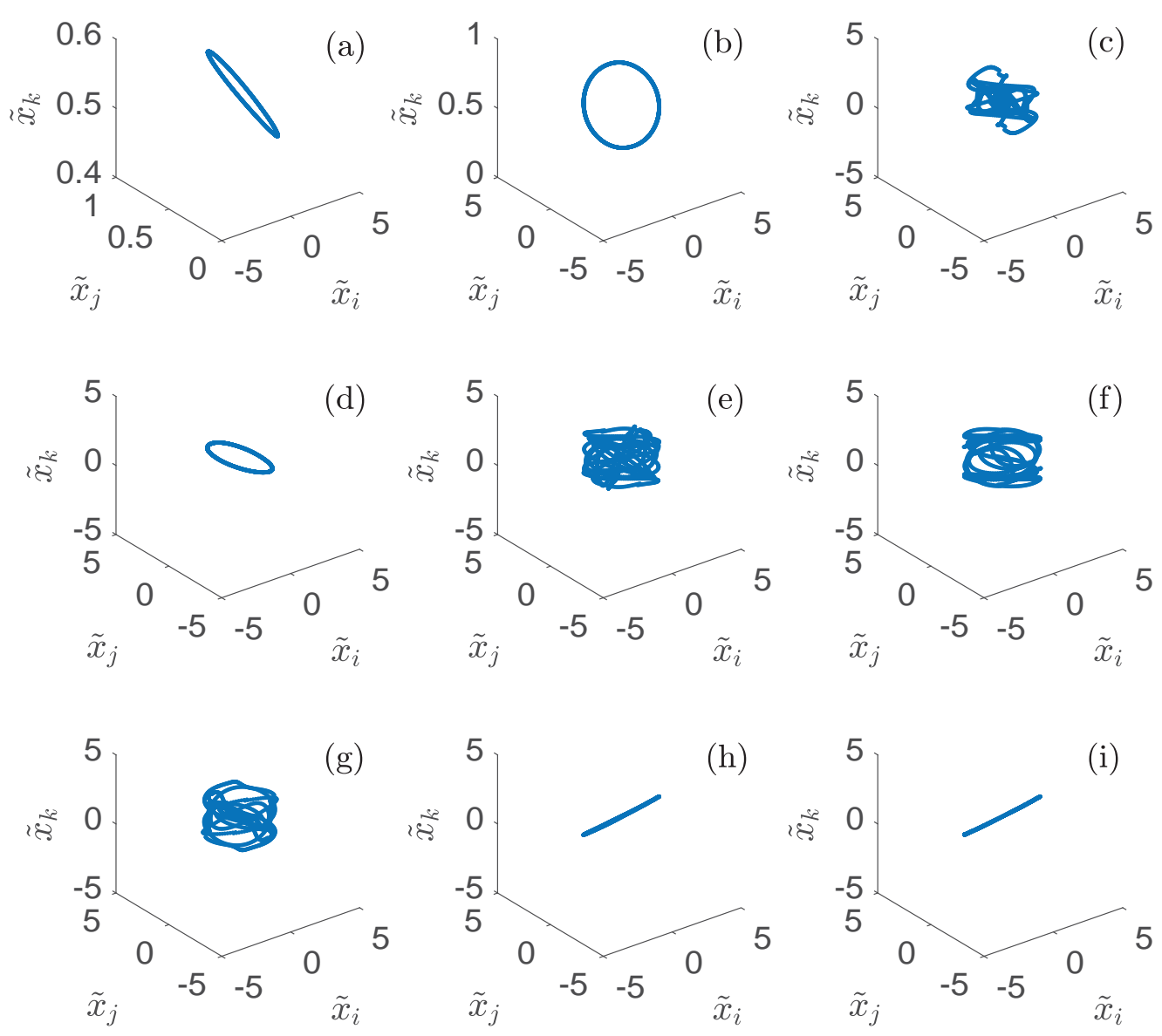

Figure 7 - Three dimensional phase plot $\left[\widetilde{x}_{11}, \widetilde{x}_{12}, \widetilde{x}_{13}\right]$ for the same solutions reported in Fig. 6, for the time window $\tau \in[1,1.5] \times 10^{4}$. The subplots (a-b-d-h-i) (solution number $[1,2,8,19,20]$ in Fig. 4) show a periodic dynamical behavior while the subplots (c-e-f-g) (solution number $[5,9,12,13]$ in Fig. 4) show irregular motion.

The dynamical behavior of the system is summarized in Fig. 8. The orbit diagram shows the projection of the time delay $\phi$ in the time span $\tau \in[1,1.5] \times 10^{4}$ versus the solution number (see Fig. 4). If the projection is composed of just "separated" single dots, the motion is periodic as it evolves with fixed time delay. When $\phi$ changes irregularly, multiple points appear thus irregular dynamics is expected. We notice that we are not changing any nonlinear parameter in the model, but only the number of oscillators excited in the very beginning, i.e. the initial conditions, and this aspect is an interesting original outcome of our model, in the best of the authors' knowledge. 


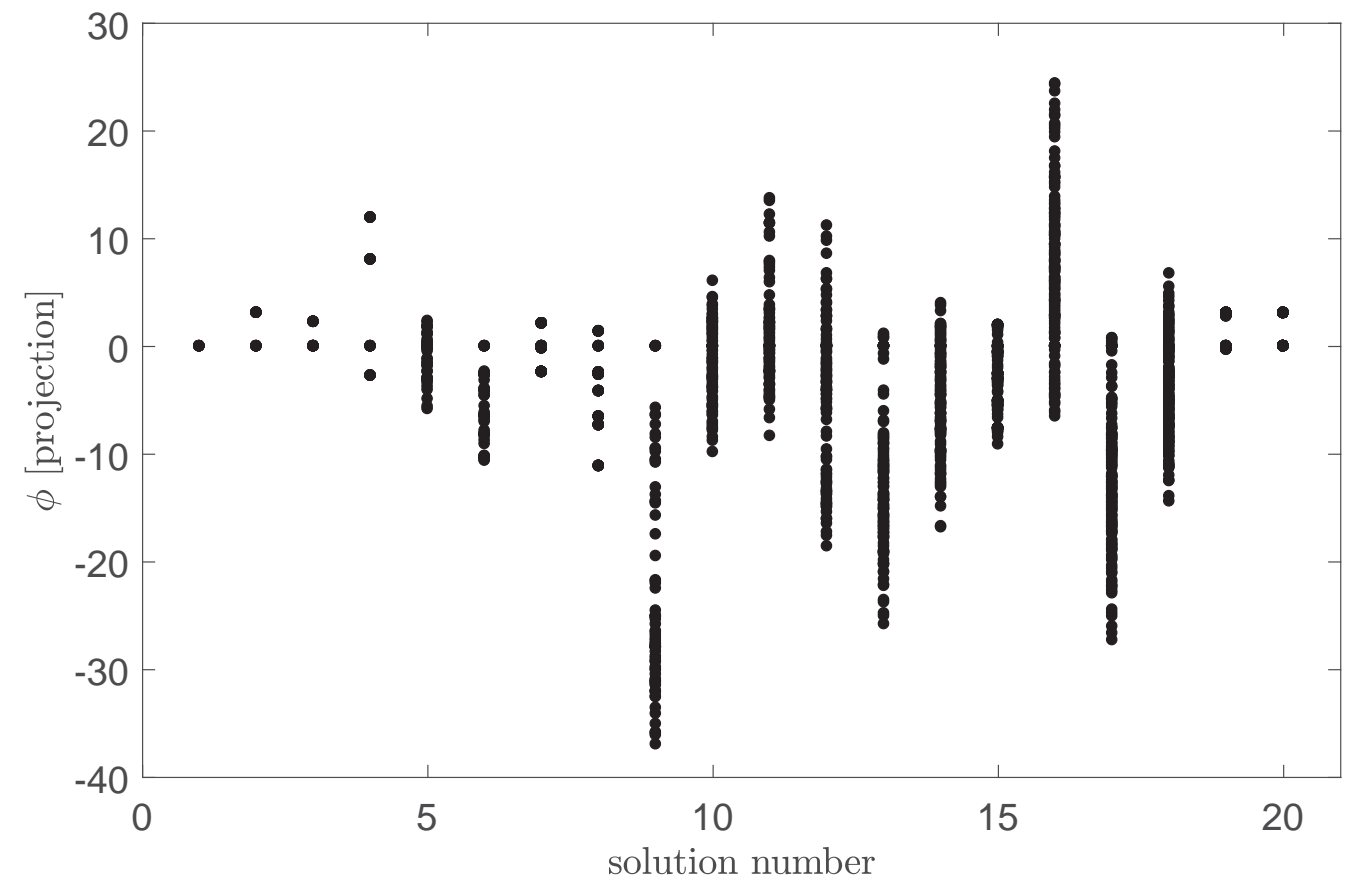

Figure 8 - Orbit diagram. On the x-axis we report the number of the oscillators which lie on the HALC, while on the y-axis is reported the projection of the time delay $\phi$ for the last 5000 integration points. The plot suggests the solutions with $[1,4] \cup[7] \cup[20]$ vibrating oscillators are periodic while the solutions $[5,6] \cup[9,18]$ exhibits an irregular dynamical behaviour.

\section{Possible localized vibrating patterns}

\subsection{Effect of the driving velocity}

We have shown in the previous paragraph the results obtained with $\widetilde{v}_{d}=$ 1.5 and with a given set of initial conditions: at $\tau=0$ we assumed $\widetilde{x}_{n}(0)=0$ for all the oscillators while varying the initial displacement $\widetilde{x}_{n}(0)$ in out-ofphase manner (8). Changing the driving velocity has an effect on the results obtained. We show in Fig. 9 the case of belt velocity $\widetilde{v}_{d}=1.65$. Comparing Fig. 9 with Fig. 8-4 it can be seen that increasing the belt velocity leads to more irregular solutions. Further in this case the number of oscillators excited by the initial conditions doesn't always match the oscillators which lie on the HALC after the transient. 

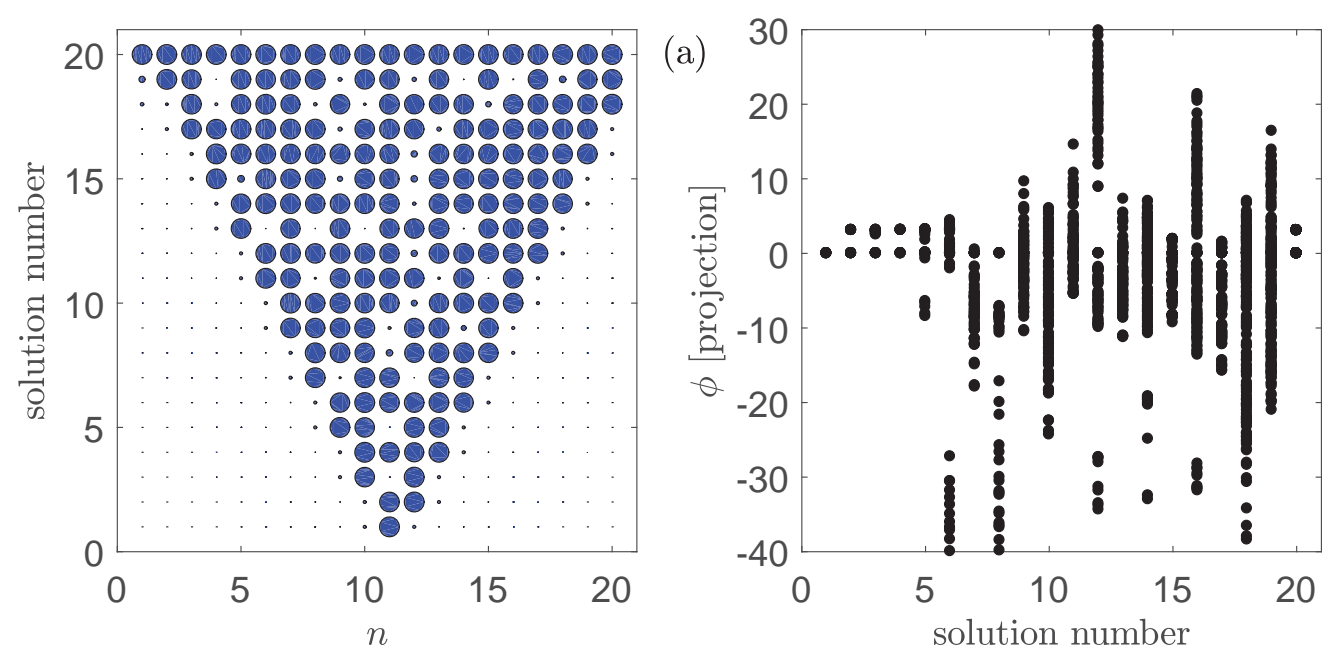

Figure 9 - (a) Localized states for $\widetilde{v}_{d}=1.65$. Each circle represents a oscillator, with the radius proportional to the amplitude of the vibration.

(b) Orbit diagram (as in Fig. 8) for the solution in the panel (a)

\subsection{Effect of the initial conditions}

A completely different result in term of localized states pattern can be obtained changing the initial conditions. Previously we have always assumed $\widetilde{x}_{n}(0)=0$ for all the oscillators and varied the initial displacement $\widetilde{x}_{n}(0)$ in out-of-phase manner (8). Naturally this is not the only possibility, as more in general, all the $2 N$ initial conditions can be arbitrarily defined leading to much more solution patterns with respect to what we have shown here. As an example in Fig. 10 we show the case of driving velocity $\widetilde{v}_{d}=1.75$ and the following initial conditions:

$$
\begin{array}{llrl}
\dot{\widetilde{x}}_{n}(0) & =0.56 & & \forall n \\
\widetilde{x}_{n}(0) & =\widetilde{F}\left(\widetilde{v}_{d}\right) & & n \text {-th oscillator is not excited } \\
\widetilde{x}_{n}(0) & =2 \times(-1)^{n}+\widetilde{F}\left(\widetilde{v}_{d}\right) & & n \text {-th oscillator is excited }
\end{array}
$$

In particular, we used a constant positive velocity for all the oscillators which, coupled with the out of phase initial conditions on the displacements, introduces an asymmetry in the system which reflects in the peculiar pattern of vibrating oscillators shown in Fig. 10 (a). Also notice that, in contrast with previous results, all the solutions obtained (peraphs except the $18^{\text {th }}$ ) are periodic (see Fig. 10 (b)). 

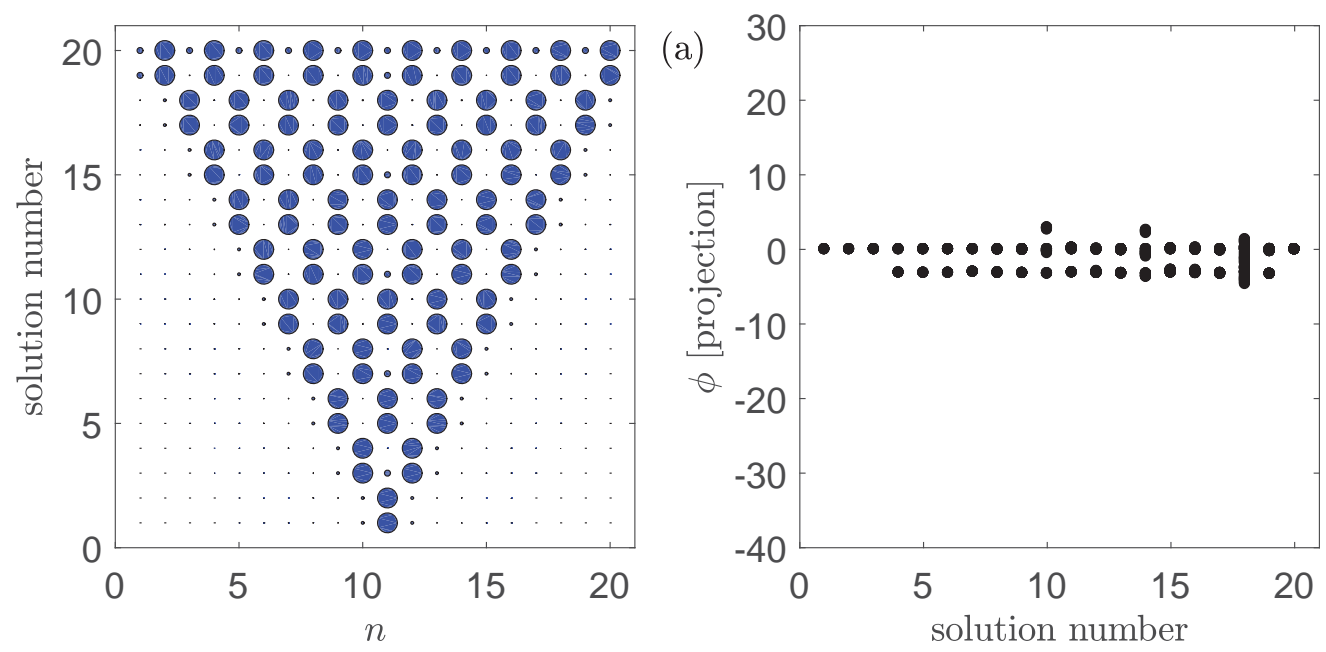

Figure 10 - (a) Localized states for $\widetilde{v}_{d}=1.75$ and $\widetilde{x}_{n}(0)>0(\forall n)$. Each circle represents a oscillator, with the radius proportional to the amplitude of the vibration. (b) Orbit diagram (as in Fig. 8) for the solution in the panel (a)

\section{Conclusions}

We have studied a cyclic symmetric oscillator chain with weak nearest neighbour coupling excited by a frictional belt driven at a constant velocity $\widetilde{v}_{d}$. Friction at the interface has been described using an exponential decaying friction law. We used the belt velocity as a bifurcation parameter and showed that in a certain interval multiple localized vibration states exist, where the vibration localizes on few oscillators. The bifurcation diagram suggests an underlying snaking pattern, as it has now been observed in many other fields of physics. We have shown that for a given set of control parameters changing the initial conditions can lead to very different localized states, ranging from localised regular to localised irregular patterns. In light of these results, the well-known lack of repeatability and "capriciousness" of friction-induced vibration problems, can be interpreted as the outcome of the multiple regular/irregular states, which characterize friction-excited systems. The results are relevant for all engineering systems that experience frictioninduced vibrations, particularly if assembled modularly and with weak coupling. Further work is required to understand how this nonlinear mechanism of localization caused by friction can interact with linear localization phenomena (Anderson localization) occurring when the underlying linear system is not homogeneous, as in all practical applications. 


\section{Acknowledgements}

A.P. and N.H. are thankful to the DFG (German Research Foundation) for funding the project HO 3852/11-1. M.S. is thankful to the DFG (German Research Foundation) for funding the project HO 3852/12-1.

\section{References}

[1] K. Y. Sanliturk, D. J. Ewins, A. B. Stanbridge. Underplatform dampers for turbine blades: theoretical modelling, analysis and comparison with experimental data. ASME 1999 international gas turbine and aeroengine congress and exhibition. American Society of Mechanical Engineers, 1999.

[2] C. M. Firrone, S. Zucca,. Underplatform dampers for turbine blades: The effect of damper static balance on the blade dynamics. Mechanics Research Communications, 36(4) (2009) 515-522.

[3] A. Papangelo, M. Ciavarella. Optimal normal load variation in wedgeshaped Coulomb dampers. The Journal of Strain Analysis for Engineering Design, 51(4), (2016) 279-285.

[4] L. Pesaresi, L. Salles, A. Jones, J.S. Green, C.W. Schwingshackl. Modelling the nonlinear behaviour of an underplatform damper test rig for turbine applications. Mechanical Systems and Signal Processing, 85, (2017) 662-679.

[5] L. Pesaresi, L. Salles, R. Elliott, A. Jones, J. S. Green, C. W. Schwingshackl. Numerical and experimental investigation of an underplatform damper test rig. In Applied Mechanics and Materials (849), (2016) 1-12

[6] M. Tiedemann, S. Kruse, N. Hoffmann, Dominant damping effects in friction brake noise, vibration and harshness: the relevance of joints. Proc. Inst. Mech. Eng. D J. Automob. Eng. 229(6) (2014) 728-734

[7] M. Stender, A. Papangelo, M. Allen, M. Brake, C. Schwingshackl, M. Tiedemann, M. Structural Design with Joints for Maximum Dissipation. In Shock \& Vibration, Aircraft/Aerospace, Energy Harvesting, Acoustics \& Optics, Volume 9, (2016) 179-187 
[8] K. Padmanahban, A. Murty, Damping in structural joints subjected to tangential loads. Proc. Inst. Mech. Eng. 205 (1991) 121-129

[9] K. Padmanahban, Prediction of damping in machined joints. Int. J. Mach. Tools Manuf. 32 (1992) 305-312

[10] A. Papangelo, M. Ciavarella. Effect of normal load variation on the frictional behavior of a simple Coulomb frictional oscillator. Journal of Sound and Vibration, 348, (2015) 282-293.

[11] J. Armand, L. Pesaresi, L. Salles, C.W. Schwingshackl. A Multiscale Approach for Nonlinear Dynamic Response Predictions With Fretting Wear. Journal of Engineering for Gas Turbines and Power, 139(2), (2017) 022505.

[12] G. Costagliola, F. Bosia, N. M. Pugno. Static and dynamic friction of hierarchical surfaces. Physical Review E, 94(6), (2016), 063003.

[13] G. Costagliola, F. Bosia, N.M. Pugno. 2-D Model for friction of complex anisotropic surfaces. arXiv preprint, (2017), arXiv:1706.08055.

[14] M. Popov, V. L. Popov, N. V. Popov. Reduction of friction by normal oscillations. I. Influence of contact stiffness. Friction, 5(1), (2017), 45-55.

[15] X. Mao, V.L. Popov, J. Starcevic, M. Popov. Reduction of friction by normal oscillations. II. In-plane system dynamics. Friction, 5(2), (2017), 194-206.

[16] S. Oberst, J. C. S. Lai, Statistical analysis of brake squeal noise. Journal of Sound and Vibration, 330(12) (2011) 2978-2994.

[17] S. Kruse, B. Stingl, J. Hieke, A. Papangelo, M. Tiedemann, , N. Hoffmann, M. Ciavarella, The Influence of Loading Conditions on the Static Coefficient of Friction: A Study on Brake Creep Groan. In Topics in Modal Analysis I, Volume 7 (2014) (149-160). Springer International Publishing.

[18] R. A. Ibrahim, Friction-induced vibration, chatter, squeal, and chaospart I: mechanics of contact and friction. Applied Mechanics Reviews, 47(7) (1994) 209-226. 
[19] R. A. Ibrahim, Friction-induced vibration, chatter, squeal, and chaospart II: dynamics and modeling. Appl. Mech. Rev, 47(7) (1994) 227-253.

[20] J. Brunetti, F. Massi, Y. Berthier. A new instability index for unstable mode selection in squeal prediction by complex eigenvalue analysis. Journal of Sound and Vibration, 377, (2016), 106-122.

[21] J. Brunetti, F. Massi, W. D'Ambrogio, Y. Berthier. Dynamic and energy analysis of frictional contact instabilities on a lumped system. Meccanica, 50(3), (2015), 633-647.

[22] U. Von Wagner, D. Hochlenert, P. Hagedorn, Minimal models for disk brake squeal. Journal of Sound and Vibration, 302(3) (2007) 527-539.

[23] U. Von Wagner, S. Schlagner, On the origin of disk brake squeal. International Journal of Vehicle Design, 51(1-2) (2009) 223-237.

[24] A. Papangelo, B. Stingl, N. Hoffmann, M. Ciavarella. A simple model for friction detachment at an interface of finite size mimicking Fineberg's experiments on uneven loading. Physical Mesomechanics, 17(4), (2014) 311-320.

[25] H. Nayfeh, D. T. Mook. Nonlinear Oscillations. New York: Wiley, 1979

[26] Yu. A. Mitropolskii, V. D. Nguyen. Applied Asymptotic Methods in Nonlinear Oscillations. Dorderecht: Kluwer, 1997.

[27] K. Popp, N. Hinrichs, M. Oestreich, Analysis of a self-excited friction oscillator with external excitation. Dynamics with Friction. Modeling, Analysis and Experiment 2 Part I (A. Guran, F. Pfeiffer and K. Popp, editors). Singapore. World Scientific, 1996.

[28] N. Hinrichs, M. Oestreich, K. Popp. On the modelling of friction oscillators, Journal of Sound and vibration 216 (1998) 435-459.

[29] A. Saha, B. Bhattacharya, P. Wahi, A comparative study on the control of friction-driven oscillations by time-delayed feedback. Nonlinear Dynamics, 60(1-2) (2010) 15-37. 
[30] H. Hetzler, D. Schwarzer, W. Seemann, Steady-state stability and bifurcations of friction oscillators due to velocity-dependent friction characteristics. Proceedings of the Institution of Mechanical Engineers, Part K: Journal of Multi-body Dynamics, 221(3) (2007) 401-412.

[31] A. Papangelo, M. Ciavarella, N. Hoffmann. Subcritical bifurcation in a self-excited single-degree-of-freedom system with velocity weakeningstrengthening friction law: analytical results and comparison with experiments. Nonlinear Dynamics, 90(3), (2017) 2037-2046.

[32] L. I. Manevitch, Y. V. Mikhlin, V. N. Pilipchuk, A.A Zevin, Normal modes and localization in nonlinear systems. A. F. Vakakis (Ed.). Kluwer Academic Publishers, 2001.

[33] A. Grolet, F. Thouverez, Vibration analysis of a nonlinear system with cyclic symmetry. Journal of Engineering for Gas Turbines and Power, 133(2) (2011) 022502.

[34] A. Grolet, F. Thouverez, Free and forced vibration analysis of a nonlinear \system with cyclic symmetry: Application to a simplified model. Journal of sound and vibration, 331(12) (2012) 2911-2928.

[35] F. Fontanela, A. Grolet, L. Salles, A. Chabchoub, N. Hoffmann, Dark solitons, modulation instability and breathers in a chain of weakly non-linear oscillators with cyclic symmetry, (2017). arXiv preprint arXiv:1704.00695.

[36] A. Papangelo, A. Grolet, L. Salles, N. Hoffmann, M. Ciavarella, Snaking bifurcations in a self-excited oscillator chain with cyclic symmetry. Communications in Nonlinear Science and Numerical Simulation, 44 (2017a) 108-119.

[37] A.R. Champneys, Homoclinic orbits in reversible systems and their applications in mechanics, fluids and optics. Physica D: Nonlinear Phenomena, 112.1 (1998) 158-186.

[38] O Thual, and S. Fauve, Localized structures generated by subcritical instabilities. Journal de Physique 49.11 (1988) 1829-1833. 
[39] C Beaume, A Bergeon, E Knobloch, Homoclinic snaking of localized states in doubly diffusive convection. Physics of Fluids, 23(9) (2011) 094102.

[40] Anderson, P.W. Absence of diffusion in certain random lattices, Phys. Rev., 109(5) (1958) 1492-1505.

[41] D. J. Ewins, The effects of detuning upon the forced vibrations of bladed disks. Journal of Sound and Vibration, 9(1) (1969) 65IN273-7279.

[42] C. H. Hodges, Confinement of vibration by structural irregularity. Journal of sound and vibration, 82(3) (1982) 411-424.

[43] R. I. Leine, D. H. Van Campen, A. De Kraker, L. Van den Steen, Stickslip vibrations induced by alternate friction models, Nonlinear Dynamics 16(1) (1998) 41-54

[44] S. Oberst, \& J. C. S. Lai. Chaos in brake squeal noise. Journal of Sound and Vibration, 330(5), (2011), 955-975.

[45] S. Oberst, \& J. C. S. Lai. Nonlinear transient and chaotic interactions in disc brake squeal. Journal of Sound and Vibration, 342, (2015), 272-289.

[46] G. Haller, \& G. Stépán. Micro-chaos in digital control. Journal of Nonlinear Science, 6(5), (1996), 415-448.

[47] G. Csernák, G. Gyebrószki, \& G. Stépán. Multi-baker map as a model of digital PD control. International Journal of Bifurcation and Chaos, 26(02), (2016), 1650023. 